\title{
Dendrimers: Synthetic Strategies, Properties and Applications
}

\author{
URVASHI SINGH, MOHAMMAD MAQBOOL DAR and ATHAR ADIL HASHMI* \\ Department of Chemistry, Jamia Millia Islamia University, New Delhi - 110025, India. \\ *Corresponding author E-mail: dr.aahashmi@yahoo.co.in \\ http://dx.doi.org/10.13005/ojc/300301
}

(Received: June 12, 2014; Accepted: August 04, 2014)

\begin{abstract}
Macromolecular chemistry is one of those fields which have become immensely diverse both in terms of synthetic chemistry as well as applications. Dendrimers are macromolecules that are inspired from nature and are a playfield for synthetic chemist. The remarkable symmetry which results in striking structures and activity of these molecules has resulted in exponential growth of researchers and research in this field. They find their application in various fields such as inorganic, catalytic, synthetic organic, biomedical chemistry and in targeted drug delivery. The review is focused on this subject we have explaining things leaving behind the typical classification system thus making it much easier to understand. This review will give the researchers some valuable insight into the topic.
\end{abstract}

Key words: Dendrimers, Catalytic, Macromolecules, Biomedical chemistry etc.

\section{INTRODUCTION}

As the human understanding of nature's building blocks evolved, chemists all over tried to incorporate natural architectural techniques into synthetic chemistry. This brought us into an era of macromolecules and polymers. A macromolecule is typically a very large molecule commonly created by polymerization of smaller subunits. Usage of the term to describe large molecules varies among the disciplines. For example, while biology refers to macromolecules as the four large molecules comprising living things, in chemistry, the term may refer to aggregates of two or more molecules held together by intermolecular forces rather than covalent bonds but which do not readily dissociate ${ }^{1}$. According to the standard IUPAC definition, the term macromolecule as used in polymer science refers only to a single molecule. For example a single polymeric molecule is appropriately described as a "macromolecule" or "polymer molecule" rather than a "polymer", which suggests a substance composed of macromolecules ${ }^{2}$.

As polymer science has evolved over the past two centuries, the number of compositions and architectures of macromolecules synthetically accessible has also grown. The unbound intelligence and innovative power of the synthetic chemists has led to a tremendous development in terms of 
ability to easily tune the size, chemistry, topology and ultimately the properties of polymers through chemicals synthesis. This inevitably led to their widespread use in a variety of technological applications. The number of published studies of macromolecular interaction has increased almost exponentially for the past 20 years and amounts to several hundred a year at present ${ }^{3}$. Synthetic macromolecules have long been utilized to modulate properties of molecules of clinical, pharmaceutical and biotechnical interest. One such macromolecular self-assemblies are hyperbranched polymers or more precisely dendrimers.

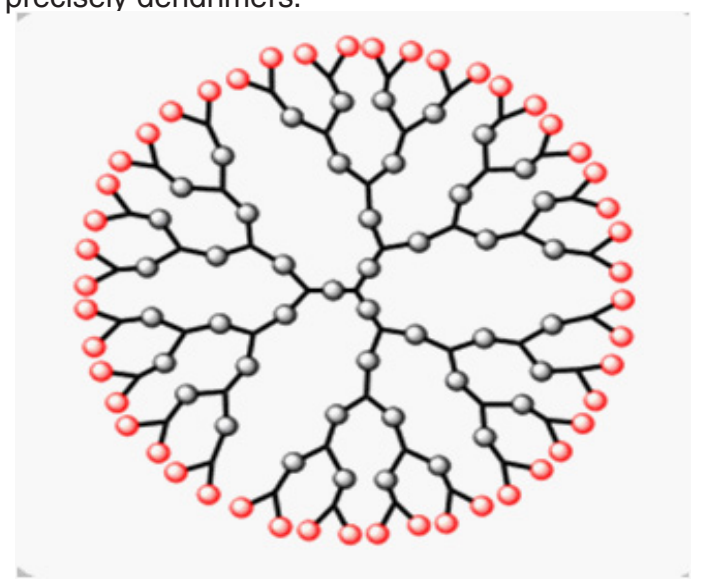

Fig. 1: Basic structure of dendrimer

Following the Watson Smith report of the reaction between phthalic anhydride or phthalic acid and glycerol in 190120, Callahan, Arsem, Dawson, Howell, and Kienle et al. ${ }^{19-21}$ studied that reaction further, obtaining results and conclusions still used today. For example, Kientle ${ }^{19}$ showed that the specific Viscosity of samples made from phthalic anhydride and glycerol was low when compared to numerous specific viscosity values given by Standinger for other synthetic linear polymers, such as polystyrene. In 1909, Baekeland ${ }^{22}$ introduced the first commercial synthetic plastics, phenolic polymers, commercialized through his Bakelite company. The cross-linked phenolic polymers are obtained by the polymerization of soluble resole precursors made from formaldehyde and phenols. Just prior to gelation, these polymers have a so-called random hyperbranched structure. In the 1940s, Flory et al. ${ }^{23-27}$ used statistical mechanics to calculate the molecular weight distribution of three-dimensional polymers with trifunctional and tetrafunctional branching
Progress over the past year clearly demonstrates that dendrimers are no longer just novel and aesthetically appealing polymers. Their unique structural features and properties make them ideally suited for both a wide range of biomedical applications and as platforms for biomimetic chemistry. Further advances in synthetic approaches will only hasten the rate at which practical uses for dendrimers are found. Hyperbranched polymers are highly branched macromolecules with threedimensional dentritic architecture. Due to their unique physical and chemical properties and potential applications in various fields from drug-delivery to coatings, interest in hyperbranched polymers is growing rapidly, as confirmed by the increase in number of publications. Since dendrimer architecture is inspired by architectural organization of the nature the molecules are more compatible with and in the living systems and with the environment. Their unique branched topologies confer dendrimers with properties that differ substantially from those of linear polymers and therefore behaviors and possible uses have and should continue to be evaluated independently from linear polymers.

This review will focus on recent progress in macromolecular dendrimer chemistry and their non medical applications. The highly branched and symmetrical molecules known as dendrimers are the most recently recognized members of the polymer family, Astruc et al., ${ }^{4}$, Smith and Diederich ${ }^{5}$, Emrick and Frechet ${ }^{6}$, Frey and Schlenk ${ }^{7}$, Hawker ${ }^{8}$, Inoue ${ }^{9}$, Meijoral and Caminerd ${ }^{10}$, Baars and Meijer ${ }^{11}$, Moore $^{12}$, Miiller et al. ${ }^{13}$, Newkome et al. ${ }^{14}$, Schliiter and Rabe ${ }^{15}$, Stoddart et al. ${ }^{16}$, Tomalia et al. ${ }^{17}$, Vogtle et al. ${ }^{18}$ and many others contributed to the development of dendrimer chemistry and the biomedical applications of dendrimers. Since these pioneering studies were done, many hundred of research groups from diverse scientific disciplines have joined the field leading to numerous advances in the synthesis, analysis and non medical application of these polymers ${ }^{19}$.

\section{History of Dendrimers}

Highly branched artificial molecules have a long and complex history. The history of hyperbranched macromolecules can be dated to the end of 19th century, when Berzelius reported the formation of a resin from tartaric acid and glycerol ${ }^{20}$. 

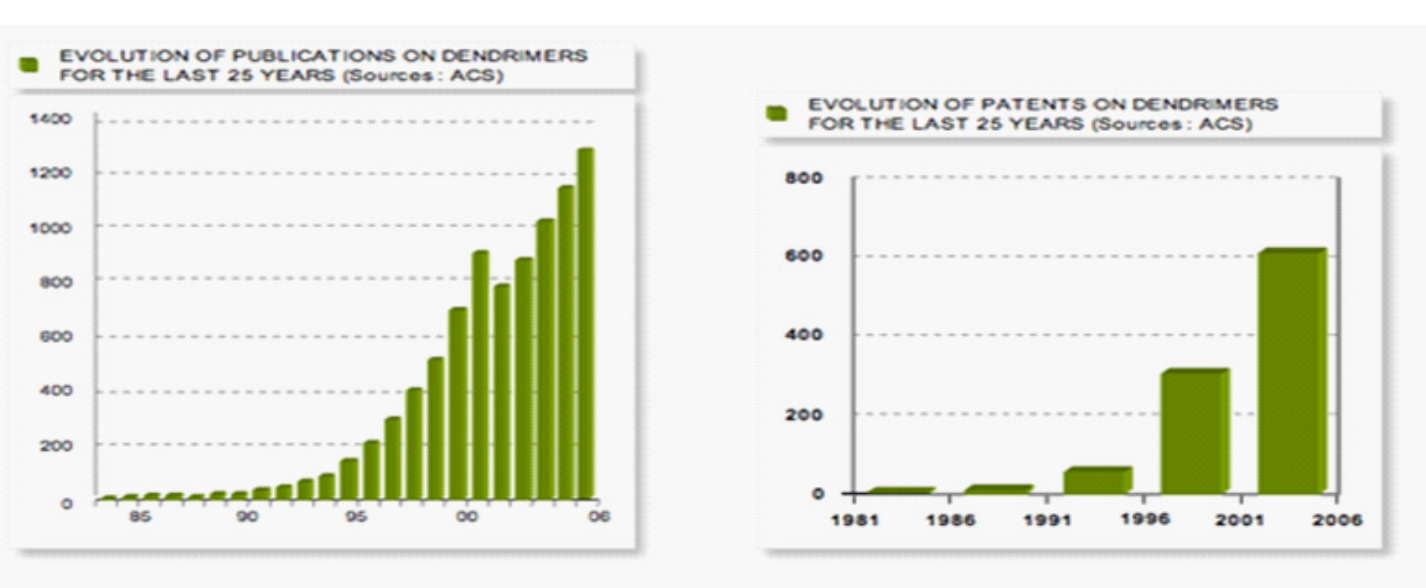

Fig. 2: The number of scientific publication as a function of publication year based on a search by ISI web of science with hyperbranched polymer as the topic [recent advances in electronic tongue, Antonio Riul Jr. ${ }^{a}$, Cléber A. R. Dantas ${ }^{b}$, Celina M. Miyazaki ${ }^{c}$ and Osvaldo N. Oliveira Jr., Analyst, 2010, 135, 2481-2495]

units in the state of gelation, and developed the 'degree of branching' and 'highly branched species' concepts. However, both the experiments and calculations mentionaed above are based on polycondensation of bifunctional monomer with trifunctional monoers, so gelation occurs when the degree of polymerization approaches the critical condition. In 1952, Flory ${ }^{28}$ developed the theory that highly branched polymers can be synthesized without the gelation by polycondensation of a monomer containing one functional group and two or more functional ones capable of reacting with each other. Finally, in 1982, Kricheldor ${ }^{29}$ obtained highly branched polyestersby copolymerization of these monomers. 'Hyperbranched polymer' was first coined by Kim and Webster ${ }^{30,31}$ in 1988 when the authors intentionally synthesized soluble hyperbranched polyphenylene. Since then, hyperbranched polymers have attracted increasing attention owing to their unique properties and greater availability as compared with dendrimers.

\section{Synthetic strategies used in Dendrimer Synthesis}

Synthesis of dendritic units is based on the principles of Click chemistry. Click chemistry was first fully described by K. Barry Sharoless of the Scripps Rsearch Institute in 2001 and describes chemistry tailored to generate substances quickly and reliable by joining small units together. Click chemistry is not a single specific reaction, but was meant to mimic nature, which also generates substances by joining small modular units.

A desirable Click chemistry reaction would:

(a) be modular

(b) be wide in scope

(c) give very high chemical yields

(d) generate only inoffensive byproducts

(e) be stereospecific

(f) be physicologically stable

(g) exhibit a large thermodynamic driving force $(>84 \mathrm{~kJ} / \mathrm{mol})$ to favor a reaction with a single reaction product

(h) have high atom economy. A distinct exothermic reaction makes a reactant "spring-loaded".

\section{The process would preferably}

1. have simple reaction conditions

2. use readily available starting materials and reagents

3. use no solvent or use a solvent that is benign or easily removed (preferably water) provide simple product isolation by nonchromatographic methods (crystallization or distillation). (fig. 3)

In chemistry a convergent synthesis is a strategy that aims to improve the efficiency of multi-step chemical synthesis, most often in organic synthesis. In linear synthesis the overall yield quickly drops with each reaction step: 

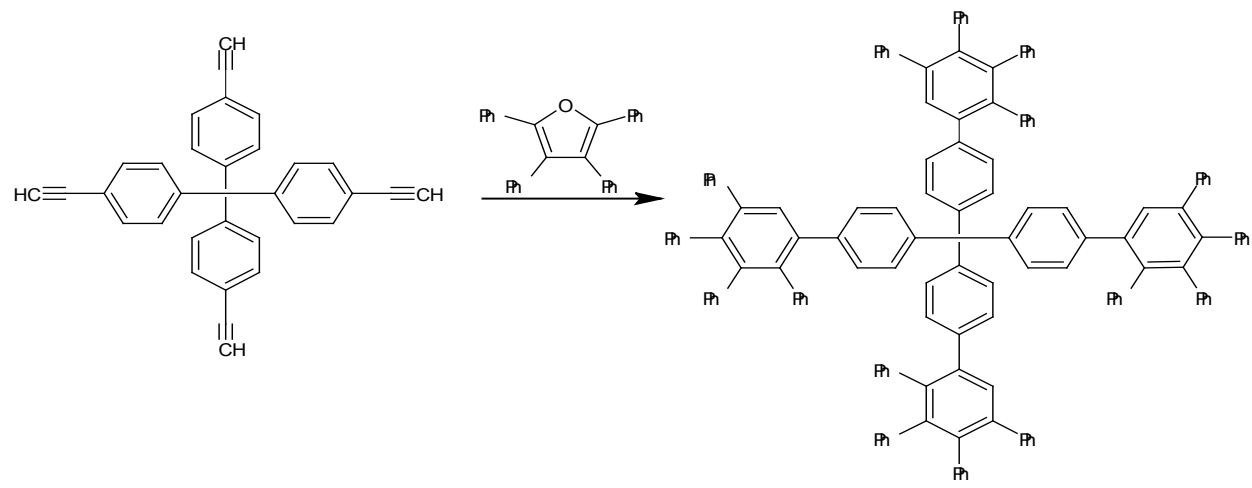

Fig. 3: Click Chemistry route

$$
\mathrm{A} \rightarrow \mathrm{B} \rightarrow \mathrm{C} \rightarrow \mathrm{D}
$$

Suppose the yield is $50 \%$ for each reaction, the overall yield of $D$ in only $12.5 \%$ from $A$. In a convergent synthesis

$$
\begin{aligned}
& A \rightarrow B(50 \%) \\
& C \rightarrow D(50 \%) \\
& B+D \rightarrow E(25 \%)
\end{aligned}
$$

The overall yield of $E$ (25\%) looks much better. Convergent synthesis is applied in the synthesis of complex molecules and involves fragment coupling and independent synthesis.

\section{Examples}

1. convergent synthesis is encountered in dendrimer synthesis where branches (with the number of generation preset) are connected to the central core.

2. proteins of upto 300 amino acids are produced by a convergent approach using chemical ligation.

3. An example of its use in total synthesis is the final step (photochemical [2+2] cycloaddition) towards the compound Biyouyanagin A. (fig. 4)

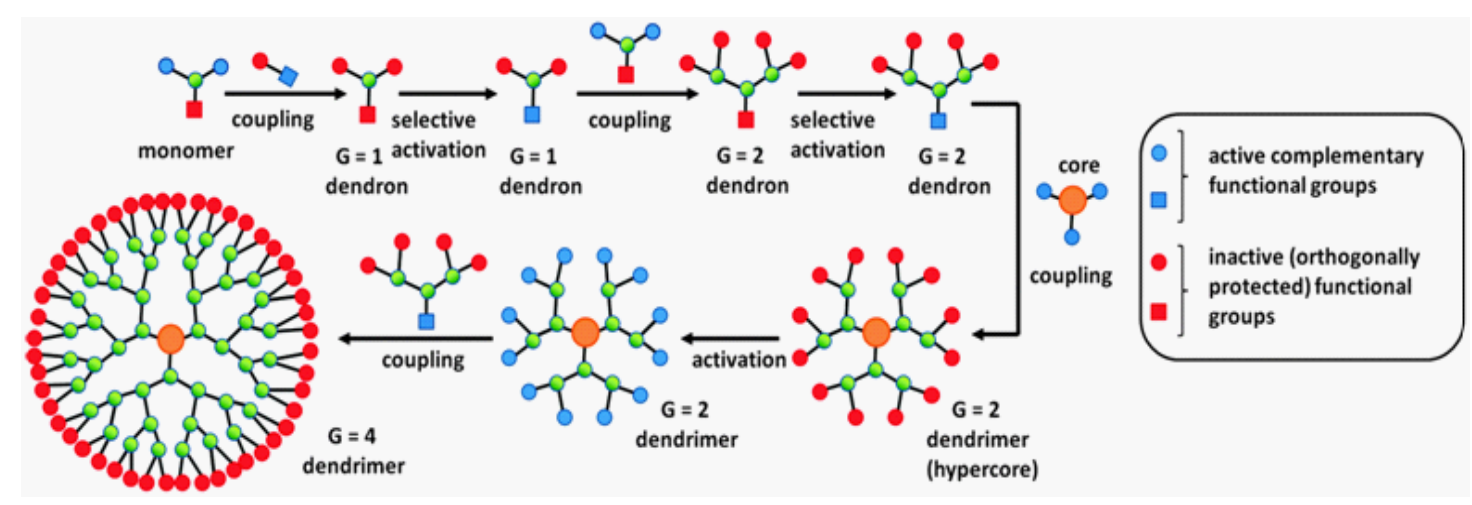

Fig. 4: Convergent Growth Method [advances in the chemistry of dendrimers, Marta Sowinska and Zofia Urbanczyk-Lipkowska, New J. Chem., 2014, 38, 2168-2203]

Divergent synthesis is a strategy that also aims to improve the efficiency of chemical synthesis. It is often an alternative to convergent synthesis or linear synthesis.

In one strategy divergent synthesis aims to generate a library of chemical compounds by first reacting a molecule with a set of reactants. The next generation of compounds is generated by further reactions with each compound in generation 1 . This methodology quickly diverge to large numbers of new compounds 
1. A generates $\mathrm{A} 1, \mathrm{~A} 2, \mathrm{~A} 3, \mathrm{~A} 4, \mathrm{~A} 5$ in generation 1

2. A1 generates $\mathrm{A} 11, \mathrm{~A} 12, \mathrm{~A} 13$ in generation 2 and so on.

An entire library of new chemical compounds for instance dendrimers can be screened for desirable properties. In another strategy divergent synthesis starts from a molecule as a central core from which successive generations of building blocks are added. A good example is the divergent synthesis of dendrimers for example where in each generation new monomer reacts to the growing surface of the sphere. (fig.5)

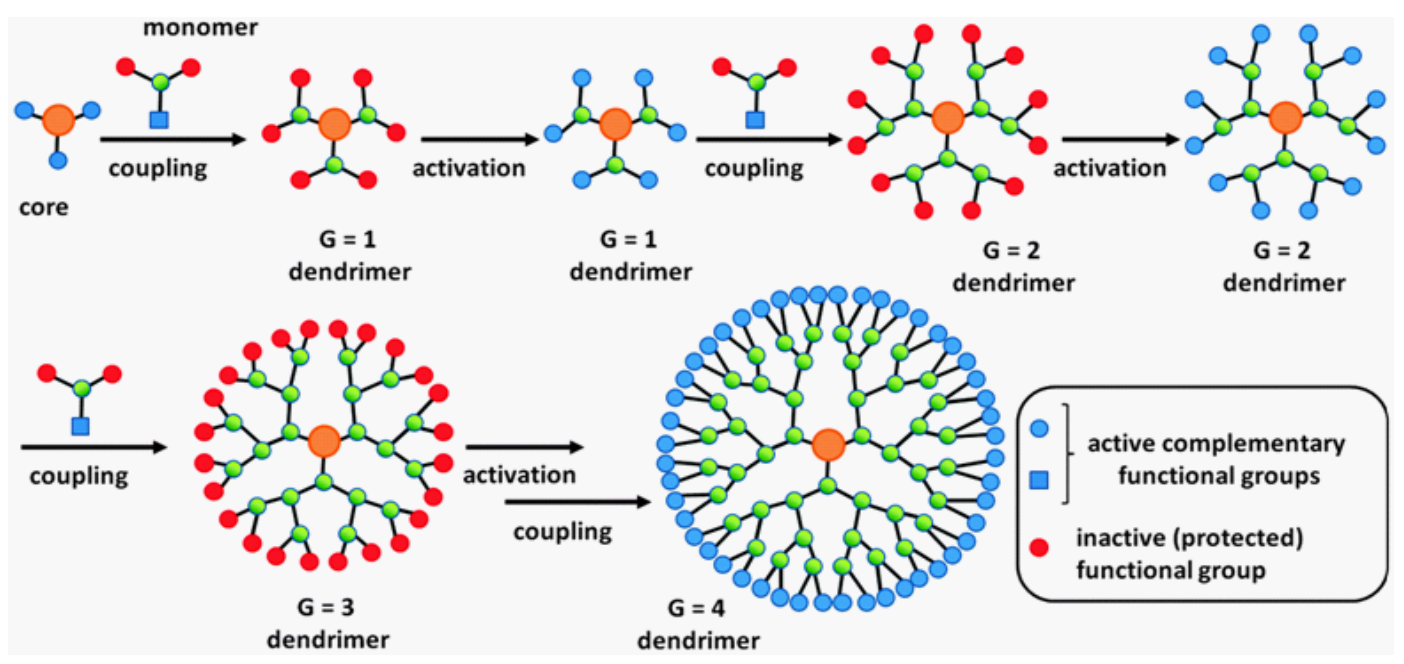

Fig. 5: Divergent Growth Method [advances in the chemistry of dendrimers, Marta Sowinska and Zofia Urbanczyk-Lipkowska, New J. Chem., 2014, 38, 2168-2203]

Diversity oriented synthesis or DOS is a strategy for quick access to molecule liberaries with an emphasis on skeletal diversity. In one such application a petasis reaction product is functionalized with propargyl bromide leading to a starting compound having 5 functional groups. This molecule can be subjected to a range of reagents yielding unique molecular skeletons in one generation.

\section{Properties}

Primarily, dendrimers properties (e.g., solubility, chemical reactivity and glass transition temperature) are controlled by nature of the end group. This is due to the exponential increase in number of end groups with increasing generation number in contrast of linear polymers which have only two end groups ${ }^{32-36}$.

In the solid state, the gradual transition in overall shape, from a more extended arrangement for lower generation dendrimers to a compact globular shape for higher generation dendrimers, causes the deviation in physical behavior of dendrimers from those of linear macromolecules. In comparison with the linear analogous, dendrimers show that the solubility is significantly increased. Due to the accessibility of the peripheral groups by globular shape, the reactivity and solubility can be controlled by the type of the peripheral groups ${ }^{32,33,37-39}$.

Dendritic volume increases cubically with generation, while dendritic molecular weight increases exponentially. This exceptional growth pattern of dendrimers leads to deviation their solution properties from those of linear molecules, especially at higher molecular weights. The physical parameter which has been used to measure these deviations is the intrinsic viscosity. Generally, the intrinsic viscosity for linear polymers increased continuously with molecular weight. On the other side, the dendrimers show very low intrinsic viscosities ${ }^{32,33,40-42}$ which reaches a maximum at a certain generation of the dendrimer. These maximum viscosities have been reported for different dendrimers (e.g., polyarylether,11a poly(propylene imine $)^{41}$ and 
PAMAM $^{42}$ ). Many of dendrimer applications depend on the availability of the large number peripheral groups (for modification, as active ligands, etc.) so it is very important to have a good understanding of peripheral groups distribution.4,5 On the contrary of highly symmetrical architectures often drown in literature, dendrimers with flexible branches can adopt different conformations. This involves backfolding of the end-groups into the interior of the molecule ${ }^{32-34}$. de Gennes and Hervet ${ }^{43}$ used the self-consistent field model to describe the dendritic architectures. In this model, monomers of each generation are assumed to be fully elongated and end-groups of the dendrimer are grouped in concentric circles around the core. This model indicates that at a certain generation (known as "de Gennes dense packing") regular growth is prohibited. It also shows that the core of the dendritic molecules has the lowest density "dense shell"34. This model can be used as good approximation to describe the highly rigid dendrimers ${ }^{44}$.
In real dendritic molecules, end-group positions depend essentially on the structure of the dendrimer. In the flexible dendrimers usually end-groups are found throughout the dendritic volume. Thus, the voids inside the dendrimer are filled up to a certain extent "dense core"32. However, when the end-groups can communicate with each other via secondary interactions including ð-ð interactions, electrostatic repulsions, hydrogenbonding interactions or hydrophobic effects, the dendritic terminal units will assemble at the periphery "dense shell"32. Based on the idea of interaction between the end groups, Meijer et. al. trapped small molecules inside the "dendritic box" of poly(propylene imine) dendrimer. The guest molecules were kept encapsulated inside the box by forming a closed shell on the surface. This shell is obtained by reacting the terminal amine with an amino acid (Lphenylalanine) $)^{32-36,45,46 .}$

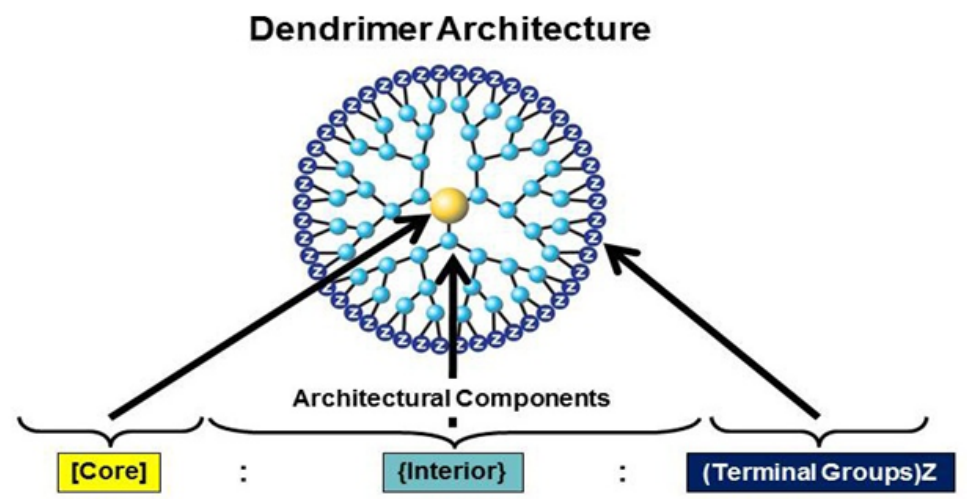

Fig. 6: Architectural components of dendrimers

The dendritic architecture contains different micro-environments (e.g., core, branches, or periphery).

In order to probe the properties of microenvironments photoactive groups introduced in the desired microenvironment. Most of studies reveal that a typical microenvironment (core environment) present inside the dendrimer which becomes more shielded from the medium at higher generations ${ }^{35,47}$. This kind of site isolation was used to mimic different natural systems by introduction of the functional groups (i.e., porphyrins) at the core. For example, Diederich et al. prepared zinc porphyrin dendrimers
(Figure 3) as a model to mimic the electron-transfer proteins (e.g., cytochrome $C)^{34,35,48-50}$.

There are now more than fifty families of dendrimers, each with unique peoperties, since the surface, interior and core can be tailored to different sorts of application. Many potential applications of dendrimers are based on their unparalleled molecular uniformity, multifunctional surface and presence of internal cavities. These specific properties make dendrimers suitable for a variety of high technology uses including biomedical and industrial applications. These applications are as follows (fig. 7): 


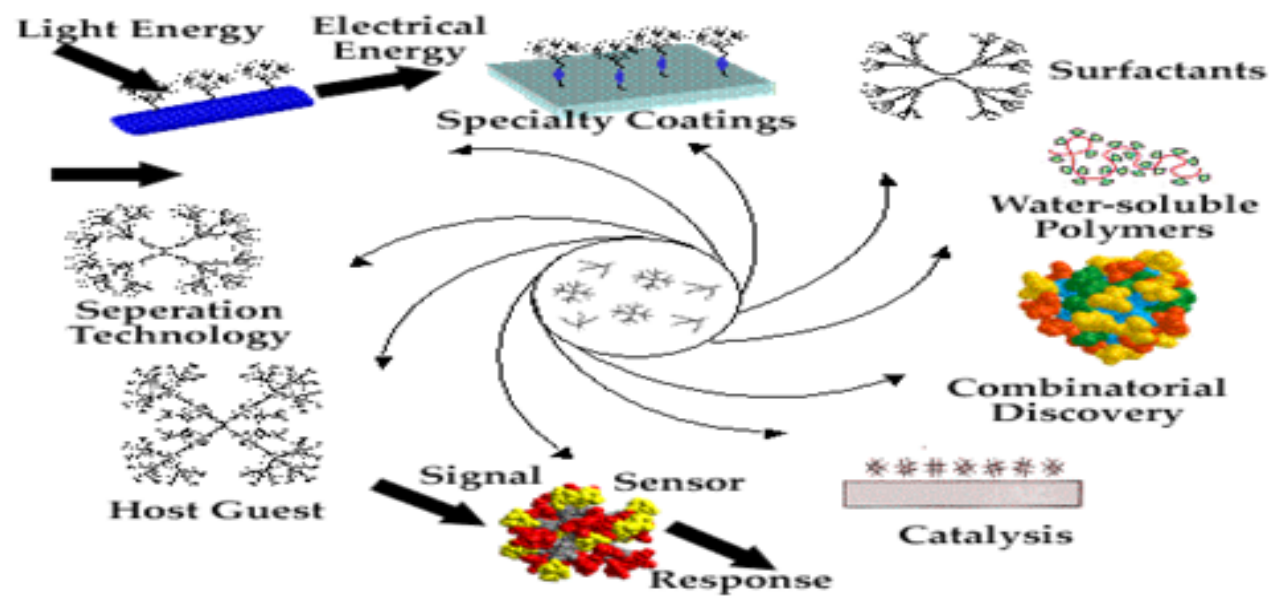

Fig. 7: Applications of dendrimers

\section{Diagnostics $^{51}$}

Paramagnetic metal chelates such as Gd(III)-N,N',N",N"'-tetracarboxymethyl-1,4,7,10tetraazacy-clododecane (Gd(III)-DOTA), Gd(III)- diethylenetri- amine pentaacetic acid (Gd(III)-DTPA), and their derivatives used as contrast agents for magnetic resonance imaging $(\mathrm{MRI})^{52}$.
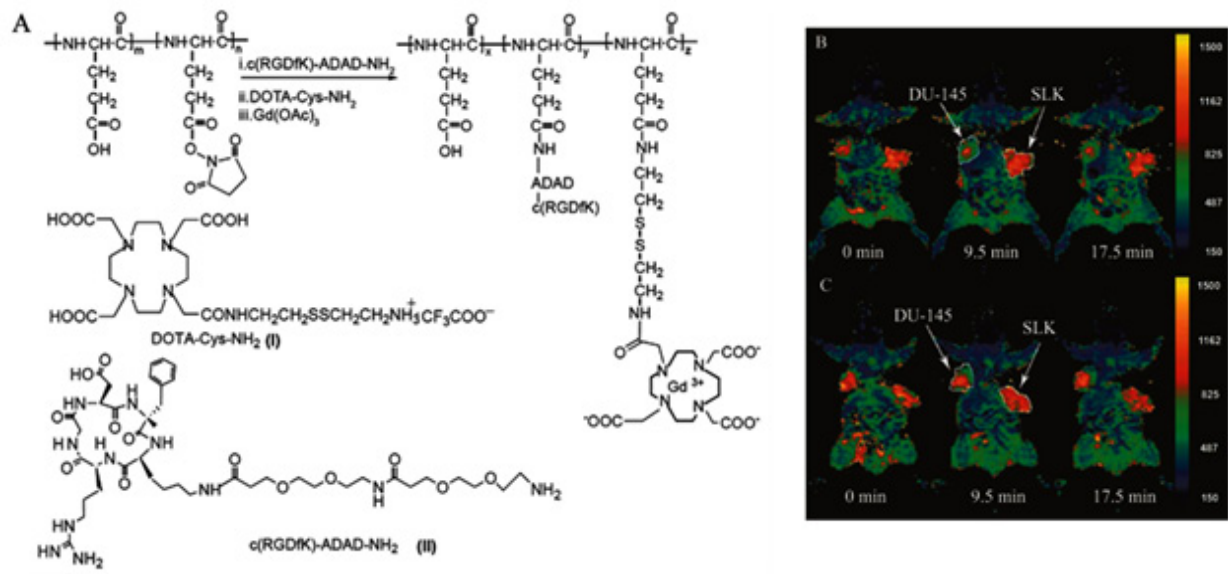

Fig. 8: Dendrimer as a contrast agent [Integrin Targeted MR Imaging Mingqian Tan, Zheng-Rong Lu, Theranostics 2011; 1:83-101]

The (Gd(III)-DTPA) conjugate (MagnevistR) (Schering $A G$ ) and is a widely used MRI contrast agent. In another approach, the conjugation of (Gd(III)-DOTA) to poly(I-glutamic acid) (molecular weight $50 \mathrm{kDa}$ ) via the biodegradable disulfide spacer cystamine was studied to find a safe and effective macromolecular MRI contrast agent. Consequently, dendrimer-based $\mathrm{Gd}(\mathrm{III})$ chelates consisting of generations 2 and 6 PAMAM dendrimers with 12 and 192 terminal surface amines conjugated to the chelating ligand 2-(4-isothiocyanatobenzyl)-6- ethyldiethylenetriamine- pentaacetic acid through a thiourea linkage were synthesized .These contrast agents exhibited excellent MRI images of blood vessels upon intravenous injection. These dendrimer polychelates were exploited for high-quality MR angiography (MRA) images up to $60 \mathrm{~min}$ post injection. DNA-dendrimers, which are constructed for routine use in high-throughput functional genomic analysis, and as biosensors for the rapid diagnosis have genetic, and pathogenesis disease ${ }^{53}$. 
Radiolabelled monoclonal antibodies with high specific activity have been Prepared by attachment of PAMAM dendrimers loaded with ${ }^{111}$ In or ${ }^{153} \mathrm{Gd}$ complexes In vivo oxygen imaging is a strategy that offers the potential for diagnosing complications from diabetes and peripheral vascular diseases, as well as the detection of tumors and the design of their therapeutic treatment ${ }^{53}$.

\section{Dendritic Catalysts / Enzymes}

The combination of high surface area and high solubility makes dendrimers useful as nanoscale catalysts. Dendrimers have a multifunctional surface and all catalytic sites are always exposed towards the reaction mixture. They can be recovered from the reaction mixture by easy ultra filtration methods ${ }^{54}$. Dendritic shells can be used to create a microenvironment favorable for catalysis or provide shielding for functional groups at the dendritic core. Because of their 'pseudo'-spherical nature and their resultant conformations the metal sites in these well-deûned polymeric catalysts should be easily accessible for substrate molecules and reagents, and therefore exhibit characteristics- fast kinetics, speciûcity and solubility ${ }^{55}$.

1. Metallodendritic catalysts

2. Catalysis with phosphine-based dendrimers

3. Catalysis with (metallo)dendrimers containing chiral ligands

4. Non-metal containing dendrimers

\section{Dendrimers as Catalyst ${ }^{56}$}

Dendritic polymers have been used in large amount as catalyst. There are two major reasons for the advantage of using dendritic polymers. One of the reasons is possibility of creating a large dendrimer with many active sites. These types of catalyst are an intermediate between heterogeneous and homogeneous catalyst which can be separated easily by filtration ${ }^{57}$. The second important reason is, there is possibility of encapsulating a single catalytic site whose activities can be enhanced by dendritic superstructure ${ }^{58}$. Dendrimers have multifunctional surface with active catalytic site. Insoluble materials can be encapsulated such as metals, and transport them into a solvent in interior of dendrimer.

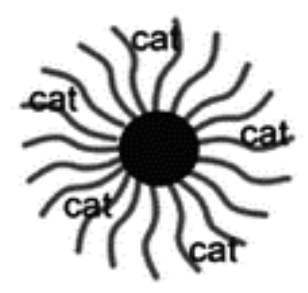

heterogeneous catalyst

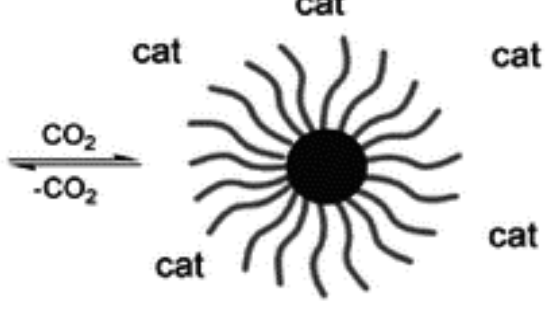

homogeneous catalyst

Fig. 9: Dendrimer as catalyst [New fluorinated functional materials, Mario Pagliaro and Rosaria Ciriminna, J. Mater. Chem., 2005, 15, 4981-4991]

\section{Industrial Processes}

Dendrimers can encapsulate insoluble materials, such as metals, and transport them into a solvent within their interior. Cooper and coworkers synthesized fluorinated dendrimers, which are soluble in supercritical $\mathrm{CO}_{2}$ and can be used to extract strongly hydrophilic compounds from water into liquid $\mathrm{CO}_{2}{ }^{60}$. This may help develop Technologies in which hazardous organic solvents are replaced by liquid $\mathrm{CO}_{2}{ }^{60}$.

\section{Dendrimers in light harvesting material}

A significant research has been of interest for designing molecules with controlled motion of charges. The use of Dendrimers is because of its multiple functionality and structural features. Moving from the periphery to the core, the functional groups decreases; which render dendrimers in light harvesting. Most of the literature report shows direction towards energy funnelling from the chromophore in the periphery to another chromophore at the core ${ }^{61}$. A study on $\sigma$-conjugated dendrimers family based on truxene and thienylethynylene were synthesised. These synthesised dendrimers show intrinsic energy gradient from periphery to the core along with broad absorption in the UV-vis range and proficient energy transfer to the lower energy centre. Hence, they are 


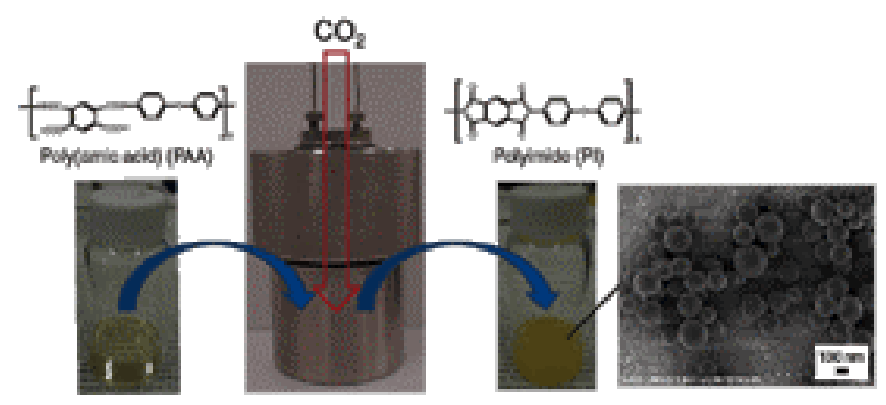

Fig.10: Dendrimer in industrial processes [Green Synthetic Method and Simple Size-control of Polyimide Nanoparticles in $\mathrm{ScCO}_{2}$

highly potential as light harvesting materials.

Dendrimers for additives, printing inks and paints

Dendrimers can be used in toners material with additives which require less material than their liquid counterparts. Xerox Corp. Patented a dry toner compound dendrimers as charge enhancing species in the form of an additive ${ }^{62}$. Using additives in printing inks, dendritic polymers ensure uniform adhesion of ink to polar and non-polar foils. Here, first the hyperbranched compounds attach themselves to the pigment particles and there are still large numbers of functional groups remaining to give adhesion to the surface of the foils. Dendritic polymers used in polyurethane paints impart surface hardness, scratch resistance, chemical resistance,

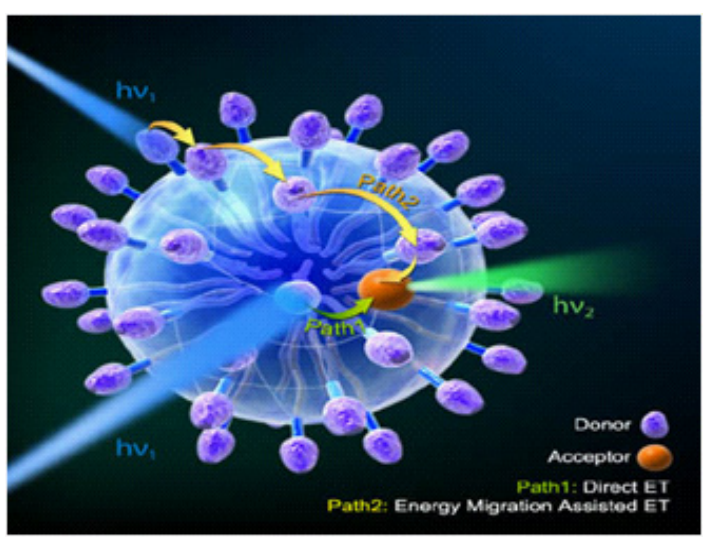

Fig. 11: Dendrimer as light harvesting material [Hui-Qing Peng, Yu-Zhe Chen, Yan Zhao, QingZheng Yang, ${ }^{*}$ Li-Zhu Wu, Chen-Ho Tung, Li-Ping Zhang, Qing-Xiao Tong, * “Artificial LightHarvesting System Based on Multifunctional Surface Cross-Linked Micelles," Angew. Chem. Int. Ed. 2012, 51, 2088-2092.] light fastness, weathering resistance as well as high gloss, because of which they are used in furniture and automotive industries. Use of Dendrimer additives in the composition of the invention is effective for altering the surface characterization of thermo plastic resin after moulding. One of example for this is polycarbonates, which are widely used as an engineering thermoplastic for providing a unique combination of toughness, stiffness, high softening temperature and processibility.

\section{Dendrimers as a separating agent}

A study of variety of compounds synthesized to determine suitability for enhancing boron rejection by reverse osmosis and nanofilteration membrane to separate born from sea water has been developed. For separation, compound must have amphiphile

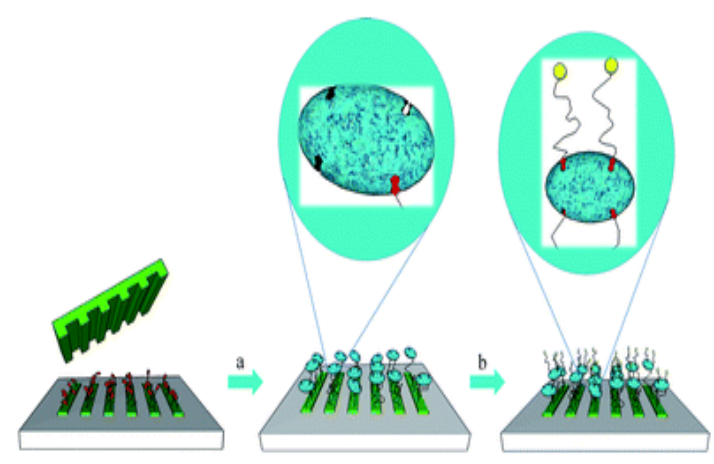

Fig. 12: Dendrimer as additive, printing inks and paints [Stamps, inks and substrates: polymers in microcontact printing 
chemical structure and form micelle in aqueous solution. As a new compound dendrimers with a high density of functional moiety, is able to form micelle structure which can be easily separated and recovered by ultra filtration membrane. These micelles provide high functional density at the surface of the particle, high surface area and ease of separation for isolation and regeneration of the compound. It was found that unmodified commercial dendrimeric compounds containing amine and hydroxyl groups are generally more effective for boron absorption. Polyamidoamine (PAMAM) dendrimers are used as chelating agents for the removal of certain metal ions from waste water ${ }^{63}$ and from contaminated soil64. Other modified chelating PAMAM and poly (propyleneimine) dendrimer are also reported to be good ligands for a various hard metal cations ${ }^{65,66}$ or can be described as "nanosponges" for the removal of Polycyclic aromatic hydrocarbons ${ }^{67}$ and other particles ${ }^{68}$.

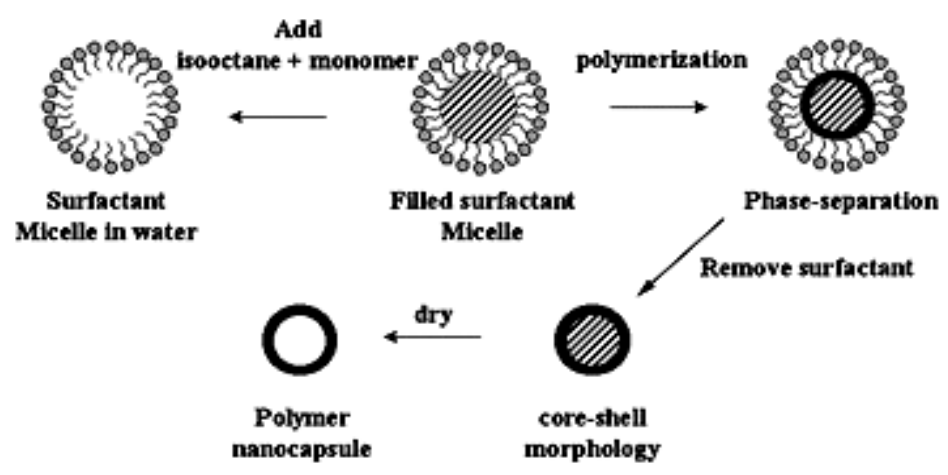

Fig. 13: Dendrimer as separating agent

\section{Future Perspective}

The following directions deserve attention in the near future:

(1) Development of new synthesis strategies and approaches based on low cost for the whole system and good control of the process and product

(2) Preparation of novel highly branched polymeric materials with hybrid architectures via covalent linking or supramolecular self-assembling (e.g. to obtain linear-hyperbranched, hyperbrachedhyperbranched, hyperbranched-polymer brush or hyperbranched-dendritic hybrid materials)

(3) Tailoring the properties of hyperbranched macromolecules by living polymerization, inorganic-organic blending, molecular encapsulation and other methods and techniques

(4) The opening of new interdisciplinary application fields for hyperbranched polymers, especiallyfields combining hyperbranched polymers with nanotechnology, micro-device technology, biology, supramolecular chemistry or single-molecule science. Finally, it can be said that not much attention has been paid to the development of dendritic units as metal capturing agents. The author proposes to undertake the research in this area, thus developing the dendritic units which can function as metal capturing ligands as well as studying the effect of metal on the functioning of known dendrimers.

\section{REFERENCES}

1. van Holde, K.E., Principles of Physical Biochemistry Prentice Hall: New Jersey, 1998, ISBN0-13-720459-0

2. Jenkins, A.; Kratochvil, P.; Stepto, R.F.T.; Suter,
U.W. Pure and Applied Chemistry, 1996, 68, 2287.

3. Kuntz, I.; Chen, K.; Sharp, K.; Kollman, P. Proc Natl Acad Sci USA. 1999, 96, 9997-10002. 
4. Astruc, D.; Blais, J,C.; Cloutet, E.; Djakovitch, L.; Rigaut, S.; Ruiz, J.; Sartor V.; Valerio, C. Top Curr Chem. 2000, 210, 229.

5. Smith, D.K.; Diederich, F. Top Curr Chem. 2000, 210, 183.

6. Emrick, T.; Fréchet, J.M.J. Curr Opin Colloid Interfac Sci. 1999, 4, 15.

7. Frey, H.; Schlenk, C. Top Curr Chem. 2000, 210, 69 .

8. Hawker, C.J. Curr Opin Colloid Interfac Sci. 1999, 4, 117.

9. Inoue, K. Prog Polym Sci. 2000, 25, 453.

10. Majoral, J.P.; Caminade, A.M. Chem Rev. 1999, 99, 845.

11. Baars, M.W.P.L.; Meijer, E.W. Top Curr Chem. 2000, 210, 131.

12. Moore, J.S. Curr Opin Colloid Interfac Sci. 1999, 4, 108.

13. Berresheim, A.J.; Müller, M.; Müllen, K. Chem Rev. 1999, 99, 1747.

14. Newkome, G.R.; He, E.F.; Moorefield, C.N. Chem Rev. 1999, 99, 1689.

15. Schlüter, A.D.; Rabe, J.P. Angew Chem. 2000, 39, 865 .

16. Matthews, O.A.; Shipway, A.N.; Stoddart, J.F. Prog Polym Sci. 1998, 23, 1.

17. Tomalia, D.A.; Wang, Z.G.; Tirrell, M. Curr Opin Colloid Interfac Sci. 1999, 4, 3.

18. Vögtle, F.; Gestermann, S.; Hesse, R.; Schwierz H.; Windisch, B. Prog Polym Sci. 2000, 25, 987

19. Kienle, R.; van, der, Meulen, P.; Petke, F. J Am Chem Soc. 1939, 61, 2258-68.

20. Kienle, R.H.; Hovey, A.G. J Am Chem Soc. 1929, 51, 509-19.

21. Kienle, R.H.; van der Meulen, P.A.; Petke, F.E. J Am Chem Soc. 1939, 61, 2268-71.

22. Odian, G. Principles of polymerization, 3rd ed. New York: Wiley; (1991), 125-32.

23. Flory, P.J. J Am Chem Soc. 1941, 63, 308390.

24. Flory, P.J. J Am Chem Soc. 1941, 63, 3091-6.

25. Flory, P.J. J Am Chem Soc. 1941, 63, 3096100.

26. Walling, C. J Am Chem Soc. 1945, 67, 441-7.

27. Flory, P.J. J Am Chem Soc. 1947, 69, 30-5.

28. Flory, P.J. J Am Chem Soc. 1952, 74, 271823.

29. Kricheldorf, H.R.; Zang, Q.Z.; Schwarx, G. Polymer. 1982, 23, 1821-9.
30. Kim, Y.H.; Webster, O.W. Polym Prepr. 1988, 29(2), 310-1.

31. Kim, Y.H.; Webster, O.W. J Am Chem Soc. 1990, 112, 4592-3.

32. Grayson, S. M.; Fréchet, J. M. Chem. Rev. 2001, 101, 3819.

33. Crespo, L.; Sanclimens, G.; Pons, M.; Giralt, E.; Royo, M.; Albericio, F. Chem. Rev. 2005 , 105,1663.

34. Bosman, A. W.; Janssen, A. M.; Meijer, E. W. Chem. Rev. 1999, 99, 1665.

35. Zeng, F.; Zimmerman, S. C. Chem. Rev. 1997, 97, 1681.

36. Klajnert, B.; Bryszewska, M. Acta Biochimica Polonica. 2001, 48, 199.

37. Wooley, K. L.; Fréchet, J. M. J.; Hawker, C. J. Polymer 1994, 35, 4489.

38. Hawker, C. J.; Malmström, E.; Frank, C. W.; Kampf, J. P. J. Am. Chem. Soc. 1997, 119, 9903.

39. Gupta, U.; Agashe, H. B.; Asthana, A.; Jain, N. K. Biomacromolecules 2006, 7, 649.

40. Mourey, T. H.; Turner, S. R.; Rubinstein, M.; Fréchet, J. M. J.; Hawker, C. J.;Wooley, K. L. Macromolecules 1992, 25, 2401.

41. de Brabander-van den Berg, E. M. M.; Meijer, E. W. Angew. Chem., Int. Ed. 1993, 32, 1308.

42. Tomalia, D. A.; Naylor, A. M.; Goddard III, W. A. Angew. Chem., Int. Ed. 1990, 29, 138.

43. de Gennes, P. D.; Hervet, H. J. Phys. Lett. 1983, 44, 351.

44. Rajadurai, M. S., Shifrina, Z. B.; Kuchkina, N. V.; Rusanov, A. L. Mullen, K. Russ. Chem. Rev. 2007, 76, 767.

45. Jansen, J. F. G. A.; de Brabander-van den Berg, E. M. M.; Meijer, E. W. Science 1994, 266, 1226.

46. Jansen, J. F. G. A.; Meijer, E.W. J. Am. Chem. Soc. 1995, 117, 4417.

47. Twyman, L. J.; King, A. S. H.; Martin, I. K. Chem. Soc. Rev. 2002, 31, 69.

48. Dandliker, P. J. Diederich, F.; Gross, M; Knobler, C. B.; Louati, A.; Sanford, E. M. Angew. Chem., Int. Ed. Engl. 1994, 33, 1739.

49. Dandliker, P. J. Diederich, F.; Gisselbrecht, J. P.; Louati, A.; Gross, M. Angew. Chem., Int. Ed. Engl. 1995, 34, 2725.

50. Dandliker, P. J.; Diederich, F.; Zingg, A.; Gisselbrecht, J. P.; Gross, M.; Louati, A.; Sanford, E. M. Helv. Chim. Acta 1997, 80, 
1773.

51. Lee, S.C. "Dendrimers In Nanobiological Devices", Dendrimers and other Dendritic Polymers Edited by Fre'chet J.M.J and Tomalia D.A., 547-554.

52. Patel, R.P. et al. Pharma Bio World, 2007, 42-52.

53. Sonke, S.; Tomalia D.A. Advanced Drug Delivery Reviews, 2005, 57, 2106 - 2129.

54. Kleij, A.W.; Ford, A.; Jastrzebskl, H.; Vankoten, G.; Dendritic Polymer Applications: Catalysts", Edited by Fre'chet J.M.J and Tomalia D.A., 485-507.

55. Kofoed, J.; Reymond, J. Current Opinion in Chemical Biology, 2005, 9, 656-664.

56. Patel, H.N.; Patel, P.M. Int. J Pharm Bio Sci. 2013, 4(2), 454-463.

57. Knapen, J.W.J.; vander Made, A.W.; de Wilde, J.C.; van Leeuwen, P.W.N.M.; Wijkens, P.; Grove, D.M.; van Koten, G. Nature 1994, 372, 659- 663.

58. Brunner, H. J.Organomet.Chem. 1995, 500, 39.

59. Cooper, A.I.; Londono, J.D.; Wignall, G.; McClain, J.B.; Samulski, E.T.; Lin, J.S.;
Dobrynin, A.; Rubinstein, M.; Burke, A.L.C.; Frechet, J.M.J.; DeSimone, J.M. Nature 1997, 389, 368-371.

60. Barbara, K.; Maria, B. Acta Biochimica Polonica 2001, 48 (1), 199-208.

61. Nantalaksakul, A.; Reddy, D. R.; Ahn, T.S.; Kaysi, R.A.; Bardeen, C. J.; Thayumanavan, S. Org. Lett. 2003, 8, 2981-2984.

62. Winnick, F.M.; Duff, J.M.; Sacripante, G.; G,Davidson A. R. Chem. Abstr. 1994, 120, 90707i.

63. Diallo, M.; Christie, S.; Swaminathan, P.; Johnson, J.; Goddard, W.; Environmental Science and Technology 2005, 39(5), 13661377.

64. Xu, Y.H.; Zhao, D.Y. Environ. Sci. Technol. 2005, 39, 2369-2375.

65. Cohen, S. M.; Petoud, S.; Raymond, K. N.; Chem. Eur. J. 2001, 7(1), 272 - 279.

66. Rether, A.; Schuster, M.; Reactive and Functional Polymers 2003, 57, 13-21.

67. Arkas, M.; Tsiourvas, D.; Paleos, C.M. Chem. Mater. 2003, 15, 2844-2847.

68. Pistilis, G.; Malliaris, A. Langmuir 2002, 18, 246-251. 